\title{
Steady-State Radial Flow Modeling through the Production Well in the Confined Aquifer of Monzoungoudo, Benin
}

\author{
François de Paule Codo ${ }^{1,2^{*}}$ (1), Babilas Hountondji' ${ }^{2}$, Martin P. Aina ${ }^{1}$ \\ ${ }^{1}$ Laboratory for Water Sciences and Technology, Department of Water and Sanitation, National Water Institute, \\ University of Abomey-Calavi, Cotonou, Benin \\ ${ }^{2}$ Physical Mathematics and Applications, University of Abomey-Calavi, Cotonou, Benin \\ Email: ^fdepaule2003@yahoo.fr, babilassrock@yahoo.fr, marnickson@yahoo.fr
}

How to cite this paper: de P. Codo, F., Hountondji, B. and Aina, M.P. (2019) Steady-State Radial Flow Modeling through the Production Well in the Confined Aquifer of Monzoungoudo, Benin. Open Journal of Fluid Dynamics, 9, 107-118.

https://doi.org/10.4236/ojfd.2019.92008

Received: April 6, 2019

Accepted: June 9, 2019

Published: June 12, 2019

Copyright $\odot 2019$ by author(s) and Scientific Research Publishing Inc. This work is licensed under the Creative Commons Attribution International License (CC BY 4.0).

http://creativecommons.org/licenses/by/4.0/

\begin{abstract}
This study aims to develop a mathematical analysis for one-dimensional modeling of a radial flow through a production well drilled in a confined aquifer, in the case of steady-state flow conditions. An analytical solution has derived from that expression for estimation of drawdowns according to different flowrates. Through that process, the evaluation of static pressure, the calculation of hydraulic charge due to the waterflow through the well is evaluated, the drawdowns curves are drawn and at last, the obtained curves are analyzed. The curves obtained for the different flow rates have an asymptotic direction, the axis of the hydraulic charges. The variation of the hydraulic charge depends on the radial distance for different flow rates. The P point, is a common point of all curves obtained for different production flowrates in the well. This point is where the well production flowrate is optimum for the optimal hydraulic charge.
\end{abstract}

\section{Keywords}

Confined Aquifer, Production Well, Radial Flow, Steady-State Flow, Drawdown, Cone of Depression

\section{Introduction}

Groundwater flows from the interconnections of aquifers to the producing well by radial flow (waterflow between aquifers and wells) and obeys to the physical phenomenon based on the relevant physical principles: Darcy's law and mass balance, (Equations (1) and (2) respectively) which are fundamental equations 
for developing theories in groundwater flow; they are:

Darcy's law:

$$
v=-K \nabla h
$$

and the mass balance

$$
\operatorname{div}-(K \nabla h)=0
$$

where $K=\frac{k \cdot \rho_{w} \cdot g}{\mu}$ and $h=z_{h}+\frac{p}{\rho_{w} \cdot g}$.

This developed theory of flow modeling process is applicable to several areas of flow mechanics, i.e. geothermal reservoirs, hydrodynamics, and mining process.

During the waterflow through the well, the hydraulic charge in the confined aquifer varies from the hydraulic charge $h_{R}$, due to the well-range $R$, to the hydraulic charge $h_{W}$ due to the radius of the well a. The present study develops first, the calculation of the drawdown, therefore, the drawdown curve (cone of depression) in the potentiometric surface around the producing well basing on Equation (1), considering that the flow regime in confined aquifer is steady-state, the hydraulic charge at any point of the aquifer remains constant, i.e. does not change with the time; thus $(\partial h / \partial t)=0$; and the fluid velocity is independent of the time. The measured data at each time (the well-range depended variable), is a point of the drawdown curve (cone of depression), in the potentiometric surface created by the hydraulic charge (in the well). The developed mathematical model determines the expression of the static pressure which can be expressed by the pressure in the well and the hydraulic charge in the piezometer (the difference between the static level and the dynamic pumping water level) and defines the variation of the drawdown cone in the potentiometric surface. The model aims to calculate the different values of the hydraulic charge in function of the radial distance ( $r$ ), with $a \leq r \leq R$, according to different values of flowrate at the wellhead.

Secondly, it develops a case study of the appearance of groundwater drawdown curves and analyzes through the developed mathematical model, their variation according of the wellhead flowrates during the water production process and their different curves.

\section{Position of the Problem}

The objective of the present paper is to determinate the static pressure of the confined aquifer and function of the pressure at the bottom of the drilling, and then simulate the variation of the hydraulic charge of the groundwater in function of the well action radius $R$, and finally to simulate mathematically the potentiometric surface of the depression cone created by the variation of the radial distance $(r)$ and the variation of the flow rates $(Q)$ at the wellhead, which describes the different levels attended from the static pressure to the hydraulic pressure at the bottom of the well. Figure 1 shows the radial flow in steady-state flow conditions around the well in a confined aquifer [1]. 


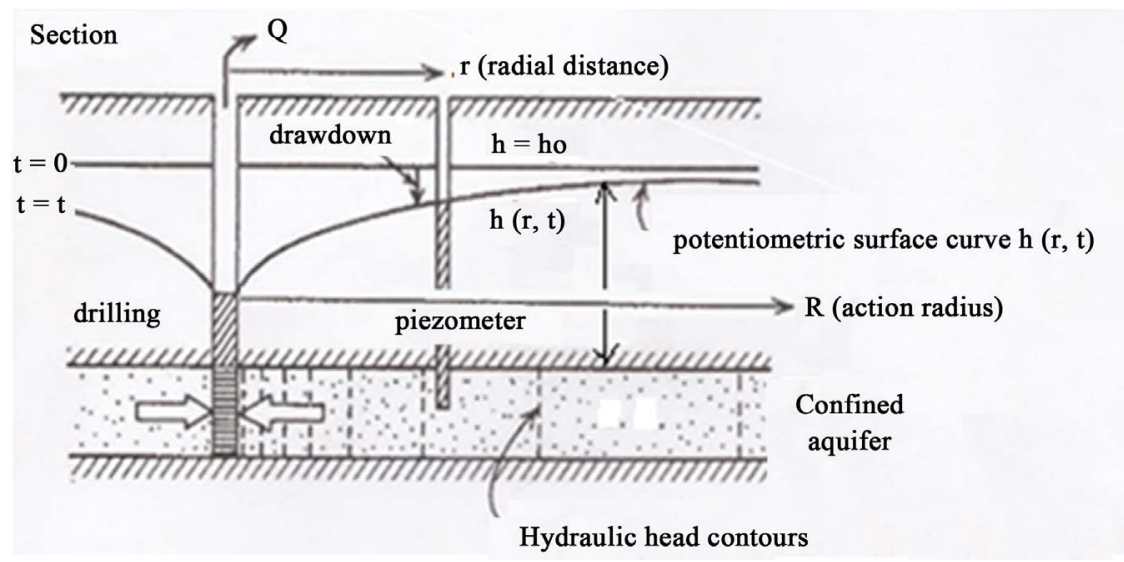

Figure 1. Steady-state radial flow in a confined aquifer.

The physical model of the problem is constituted by a confined aquifer, a porous medium which thickness is e; and a producing drilling whose flow rate is $Q$. The well cross-section is simulated to a circular section which radius is $a$. In the cylindrical coordinates system $(O, r, z) ; h_{R}$ is the hydraulic charge at the limit of the action radius $R$ in the confined aquifer, and $h_{W}$ is the hydraulic charge of the well; the reservoir, the porous medium which thickness is $e$; and the producing well which flowrate is $Q$ and $h_{R}$ are the hydraulic charge at the limit of the well-range $R$ in the reservoir [1].

The well-range is the locus point for a part of potentiometric surface curve where, the pressure does not influence the potentiometric surface in the reservoir during the groundwater exploitation, comparing to its axis, and the value of the depression cone (pumping-out), i.e. the drawdown is not available; $\left(h_{R}-h_{w}\right)=0$.

Figure 2 shows the variation of the hydraulic charge $h(r)$ in function of the radial distance.

\section{Materials and Method}

The following assumptions for the governing equations are the following:

- the porous medium is isotropic, homogenous;

- permeability, porosity $(\phi)$, hydraulic conductivity $(K)$, and productive thickness (e), are supposed to be constant;

- the density $(\rho)$, the dynamic viscosity $(\mu)$ and the cinematic viscosity $(\mathfrak{V})$ of the groundwater are considered as constants.

The water is produced in accordance to Figure 1 with the well diameter is $(D)$, the drilling depth is $(H)$ and its flow rate is $(Q)$.

The boundary conditions for the governing equations are the following.

\subsection{Governing Equations}

The governing equations are [1] [2]:

The generalized law of Darcy: 


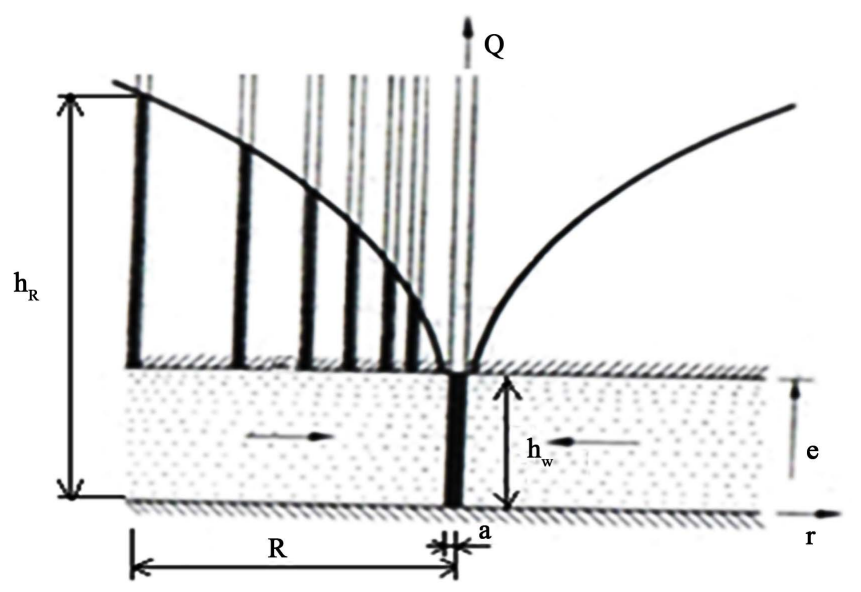

Figure 2. Variation of the drawdown curve in the potentiometric surface of the hydraulic charge from $h_{R}$ to $h_{w}$ in function of the radial distance $(a \leq r \leq R)$.

$$
v=-K \nabla h \text { with } K=\frac{k \cdot \rho_{w} \cdot g}{\mu}
$$

in the case of an incompressible fluid, $h=z_{h}+\frac{p}{\rho_{w} \cdot g}$

The continuity equation:

$$
\frac{\partial}{\partial t}(\phi)+\operatorname{div}(v)=0
$$

The diffusivity equation:

$$
\frac{\partial(\phi)}{\partial t}+\operatorname{div}(-K \nabla h)=0
$$

Considering the porosity $\phi$ independent of time, the porous medium non-deformable, taking only the hydrodynamic part and the temperature constant, $\rho$ depending only on $p$, we got:

$$
\frac{\partial(\phi)}{\partial t}=\frac{\partial(\phi)}{\partial p} \frac{\partial p}{\partial t}=\frac{s}{\rho \cdot g} \frac{\partial p}{\partial t}
$$

where $s=\rho_{w} g \frac{\partial(\phi)}{\partial p}$ is the storage-specific coefficient which shows the ability of the porous medium to release fluid when increasing the pressure. By introducing Equation (6) in Equation (4), we obtain:

$$
\frac{s}{\rho_{w} \cdot g} \frac{\partial p}{\partial t}+\operatorname{div}(-K \nabla h)=0
$$

where $h=\frac{p}{\rho_{w} g}+z_{h}$ and $\frac{\partial h}{\partial t}=\left(\frac{\partial p}{\partial t} \rho_{w} g-p g \frac{\partial \rho_{w}}{\partial t}\right) \frac{1}{\left(\rho_{w} g\right)^{2}}$

Since the fluid is incompressible, thus, $\frac{\partial \rho_{w}}{\partial t}=0$; and $\frac{\partial p}{\partial t} \approx \rho_{w} g \frac{\partial h}{\partial t}$; we got the mass balance equation for an incompressible fluid in a non-deformable medium: 


$$
s \frac{\partial h}{\partial t}+\operatorname{div}(-K \nabla h)=0
$$

the diffusivity equation for the confined groundwater aquifer.

In steady flow condition, $\frac{\partial h}{\partial t}=0$ and thus:

$$
\operatorname{div}(-K \nabla h)=0
$$

This Equation (9) allows to estimate the (hydraulic charge) potentiometric surface created hydraulic charge expression $h(r)$ in function of the hydrogeological parameters. In cylindrical coordinates, the diffusivity equation can be written as follows:

$$
\frac{\partial^{2} h}{\partial r^{2}}+\frac{1}{r} \frac{\partial h}{\partial r}+\frac{1}{r^{2}} \frac{\partial^{2} h}{\partial \theta^{2}}+\frac{\partial^{2} h}{\partial z^{2}}=0
$$

As the flow is axisymmetric flow, $\frac{\partial^{2} h}{\partial \theta^{2}}=0$ and there is no horizontal flow so $\frac{\partial^{2} h}{\partial z^{2}}=0$.

Thus the previous differential equation is reduced to:

$$
\frac{\partial^{2} h}{\partial r^{2}}+\frac{1}{r} \frac{\partial h}{\partial r}=0 \text { and becomes } \frac{\mathrm{d}^{2} h}{\mathrm{~d} r^{2}}+\frac{1}{r} \frac{\mathrm{d} h}{\mathrm{~d} r}=0
$$

Therefore $\frac{1}{r} \frac{\mathrm{d}}{\mathrm{d} r}\left(r \frac{\mathrm{d} h}{\mathrm{~d} r}\right)=0$

The integration yields:

$$
r \frac{\mathrm{d} h}{\mathrm{~d} r}=C_{1} ; \quad \mathrm{d} h=\frac{C_{1}}{r} \mathrm{~d} r ; \text { and } h=C_{1} \ln r+C_{2}
$$

Using the boundary conditions:

$$
r=a ; \quad h_{w}=C_{1} \ln a+C_{2}, \quad r=R ; \quad h_{R}=C_{1} \ln R+C_{2}
$$

with $C_{1}=\frac{h_{R}-h_{w}}{\ln (R / a)}$ and $C_{2}=h_{w}-\frac{h_{R}-h_{w}}{\ln (R / a)} \ln a$

The general solution is then:

$$
h=\frac{h_{R}-h_{w}}{\ln (R / a)} \ln r+h_{w}-\frac{h_{R}-h_{w}}{\ln (R / a)} \ln a ;
$$

thus

$$
h=\frac{h_{R}-h_{w}}{\ln (R / a)} \ln \left(\frac{r}{a}\right)+h_{w}
$$

The flow velocity into the well is calculated by using Darcy's equation and taking into account the previous boundary conditions:

$$
v=-K \frac{\mathrm{d} h}{\mathrm{~d} r}=-K \frac{h_{R}-h_{w}}{\ln (R / a)} \frac{1}{r}
$$

The flow rate can be determined by evaluating the flow at any radial distance $r$ and integrating over the flow surface. Taking into account $r=a$, we get: 


$$
\begin{gathered}
v=-K \frac{h_{R}-h_{w}}{\ln (R / a)} \frac{1}{a} \\
Q=\int_{A}(v)_{r=a} \mathrm{~d} A=\int_{\theta=0}^{2 \pi}-K \ln \frac{h_{R}-h_{w}}{\ln (R / a)} \frac{1}{a} \mathrm{~d} \theta \\
Q=-2 \pi K e \frac{h_{R}-h_{w}}{\ln (R / a)}
\end{gathered}
$$

Thus

$$
h_{R}-h_{w}=\frac{Q}{2 \pi K e}\left(\ln \frac{R}{a}\right)
$$

According to Laurent et al. [3], in a steady flow, the hydrodynamic flow pressure depends on the transmissivity of the captive reservoir by the following relation $P_{h q}=\frac{\mu Q}{2 \pi e k} \ln \left(\frac{R}{a}\right)$, and they supposed that $\ln \left(\frac{R}{a}\right)=2 \pi$ can be used in this case. By replacing Equation (13) in Equation (12) we obtain:

$$
h=\frac{Q}{2 \pi K e}\left(\ln \frac{r}{a}\right)+h_{w}
$$

The hydraulic charge of the well related to the bottom pressure of well is expressed by

$$
h_{w}=\frac{p_{w}}{\rho_{w} \cdot g}
$$

According to Codo, [4] in steady flow conditions, the pressure at the bottom of the well $P_{w}$ is related to the static pressure in the reservoir $P_{s t}$ by the following relationship: $P_{w}=P_{s t}-\frac{\mu Q}{2 \pi e K} \ln \left(\frac{R}{a}\right)$; with $\ln \left(\frac{R}{a}\right)=2 \pi$; so we get:

$$
P_{w}=P_{s t}-\frac{\mu Q}{e K}
$$

Replacing Equation (16) into Equation (15), then we get:

$$
h_{w}=\frac{p_{s t}}{\rho_{w} \cdot g}-\frac{Q}{e \cdot K}
$$

with $K=\frac{k \rho g}{\mu}$; Substituting Equation (17) into Equation (14) we obtained the expression of the hydraulic charge; $h=\frac{Q}{2 \pi K e}\left(\ln \frac{r}{a}\right)+\frac{P_{s t}}{\rho_{w} g}-\frac{Q}{e K} \quad$ [4].

Thus the expression of $h$ becomes:

$$
h=\frac{Q}{2 \pi K e}\left[\left(\ln \frac{r}{a}\right)-2 \pi\right]+\frac{P_{s t}}{\rho_{w} \cdot g}
$$

and at least

$$
h=\frac{Q}{2 \pi K e}\left(\ln \frac{r}{a}\right)-\frac{Q}{e K}+\frac{P_{s t}}{\rho_{w} \cdot g}
$$

Let us remember that the hydraulic charge $h$ for an incompressible fluid is: 


$$
h=\frac{v^{2}}{2 g}+\frac{p}{\rho_{w}}+z_{h}
$$

In the porous medium, the groundwater flow can be neglected $(v=0)$; thus, $\frac{v^{2}}{2 g}=0$ and then the hydraulic charge is equivalent to the static charge:

$$
h=\frac{p}{\rho_{w} \cdot g}+z_{h}
$$

\subsection{Evaluation of the Static Pressure in the Aquifer}

The static pressure of the water at any point of the aquifer is defined with the equation of Bernoulli [5] [6]. To formulate its expression, the simplifying assumptions are the following [7]:

- The reservoir is at a constant temperature of $20^{\circ} \mathrm{C}$, and so negligible,

- The aquifer is such that the operating range $(R)$ is very large compared to its thickness (e),

- The porous medium is homogeneous, permeable and isotropic $\left(k_{h}=k_{v}=k\right)$,

- The fluid is incompressible ( $\rho_{w}=c s t e$ ),

- The flow is radial from the reservoir to the drilling under the following conditions: $\partial^{2} h / \partial \theta^{2}=0$ et $\partial^{2} h / \partial z^{2}=0$

- The flow is two-dimensional flow $(x, y)$ and $\left(v_{z}=0\right)$,

- The flow is steady $\left(\frac{\partial h}{\partial t}=0\right)$,

- The flow is conservative ( $\operatorname{div} v=0)$.

At the wellhead, the pressure can be expressed as [4]:

$$
p_{2}=p_{1}-\rho_{w} \cdot g \cdot H-\lambda \cdot \rho_{w} H \frac{v^{2}}{2 d}
$$

with the bottom pressure $p_{1}=p_{s t}-\frac{\mu Q}{2 \pi e k} \ln \left(\frac{R}{a}\right) ; \lambda=\frac{0.086}{R e^{0.2}} ; \quad R_{e}=\frac{v d}{\vartheta}$ and $v=\frac{4 Q}{\pi d^{2}}$.

\section{After transformations}

$$
p_{2}=p_{s t}-\rho_{w} \cdot g \cdot H-\frac{\mu Q}{2 \pi \cdot e \cdot K} \ln \left(\frac{R}{a}\right)-0.06642 \rho_{w} \frac{H}{d^{4.8}} Q^{1.8} \vartheta^{0.2}
$$

with the approximation of Laurent et al. [2] [8] [9]; $\ln \left(\frac{R}{a}\right)=2 \pi$, we obtained the static pressure in the reservoir:

$$
p_{s t}=p_{2}+\rho_{w} \cdot g \cdot H+\frac{\mu Q}{e \cdot K}+0.06642 \rho_{w} \frac{H}{d^{4.8}} Q^{1.8} \vartheta^{0.2}
$$

The calculation of the static pressure assumed the use of the parameters of the well and aquifer. Just before the pumping operation $(t=0)$, the hydraulic charge is uniformly distributed throughout the aquifer, the initial condition is $h(x, y, z, o)=h_{R} ; \forall(x, y, z)$. 
The two boundary conditions associated with the problem are the following:

- At the aquifer: $r=R$, the charge is $h_{R}$

- At the well: $r=a$, the charge is $h_{W}$

\subsection{Hydrodynamic Parameters of the Reservoir}

From the test pumping carried out on the aquifer of Monzoungoudo by DGEau, we obtained some values of hydrodynamic parameters whose are the following:

- Temperature of the well is constant at $20^{\circ} \mathrm{C}$,

- Water density $\rho_{W}=1000 \mathrm{~kg} / \mathrm{m}^{3}$.

- Dynamic viscosity $\mu=10^{-3} \mathrm{~Pa} \cdot \mathrm{s}$.

- Kinematic viscosity $\vartheta=10^{-6} \mathrm{~m} / \mathrm{s}$.

\subsection{Geometric and Hydrodynamic of the Well of Monzoungoudo}

Geometric and hydrodynamics data of Monzoungoudo well, are provided and collected in Table 1 (DGEau, Benin). The well of Monzoungoudo is drilled across all the geological formations of the study area. The aquifer is deep respectively from the roof at $201 \mathrm{~m}$ to the wall at $244.18 \mathrm{~m}$; so the thickness of the aquifer is about $43.18 \mathrm{~m}$.

From the pumping test of the aquifer, the hydrodynamic parameters obtained are the followings:

- Permeability: $2.28 \times 10^{-4} \mathrm{~m} / \mathrm{s}$.

- Average porosity: $30 \%$.

And the following table shows the geometric and the hydrodynamic characteristics of the Monzoungoudo well.

\section{Results and Analysis}

The following Figure 3(a) and Figure 3(b) reveal the evolution of the hydraulic charge as a function of radial distance when water flowrates vary; $0.002 \otimes Q Q$ 0.005 in Figure 3(a) and $0.006 \odot Q \otimes 0.009$ in Figure 3(b).

The flowrates vary from $0.002 \mathrm{~m}^{3} / \mathrm{s}$ to $0.009 \mathrm{~m}^{3} / \mathrm{s}$ and the representative curves are simulated to a logarithmic function. The common point of the curves are determined by transforming the Equation (13), and then, we obtain the following relation:

Table 1. Values of geometric and hydrodynamic characteristics of Monzoungoudo well (source: DGEau, Benin).

\begin{tabular}{cccc}
\hline Symbols & Description & Values & Units \\
\hline$H$ & Depth of the well & 244.18 & $\mathrm{~m}$ \\
$Q$ & Diameter of the well & 0.126 & $\mathrm{~m}$ \\
$g$ & Flow rate & 2000 & $\mathrm{~cm} 3 / \mathrm{s}$ \\
$p_{2}$ & Pressure at the head of the well & 9.81 & $\mathrm{~m} / \mathrm{s} 2$ \\
$\varepsilon$ & Absolute roughness of the pipe & 4.16 & $\mathrm{bar}$ \\
\hline
\end{tabular}




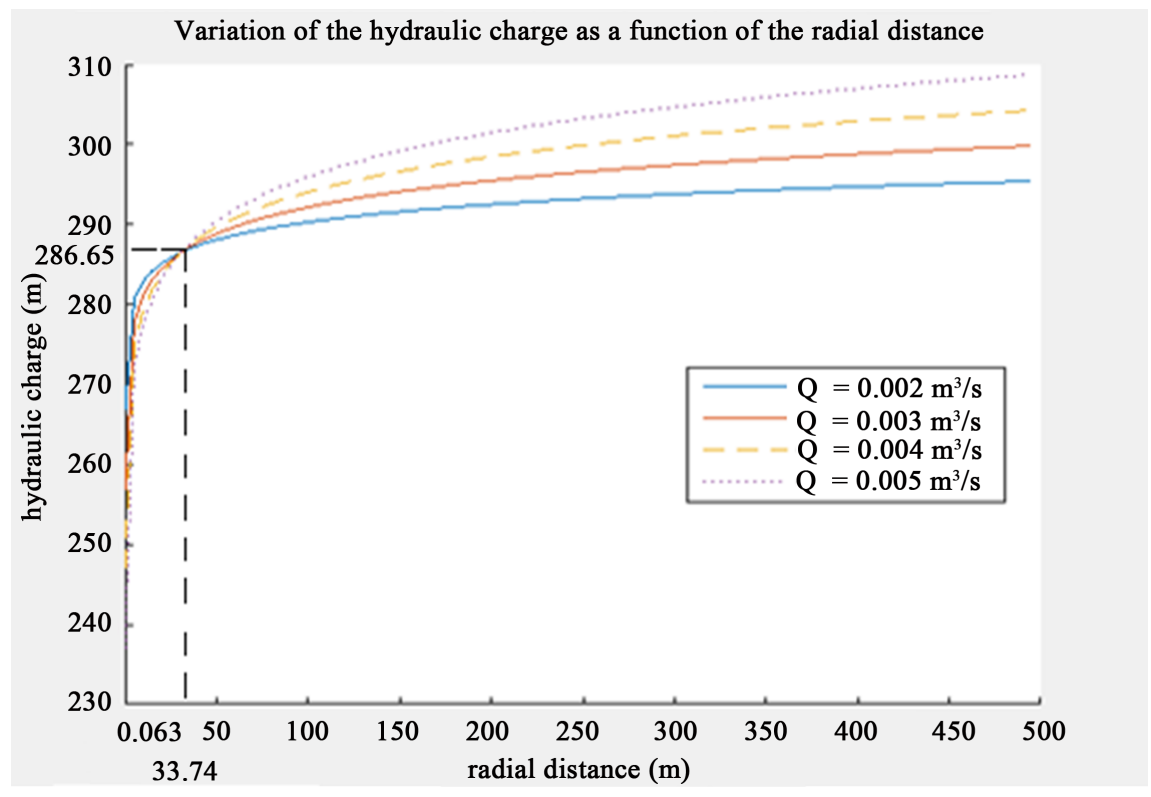

(a)

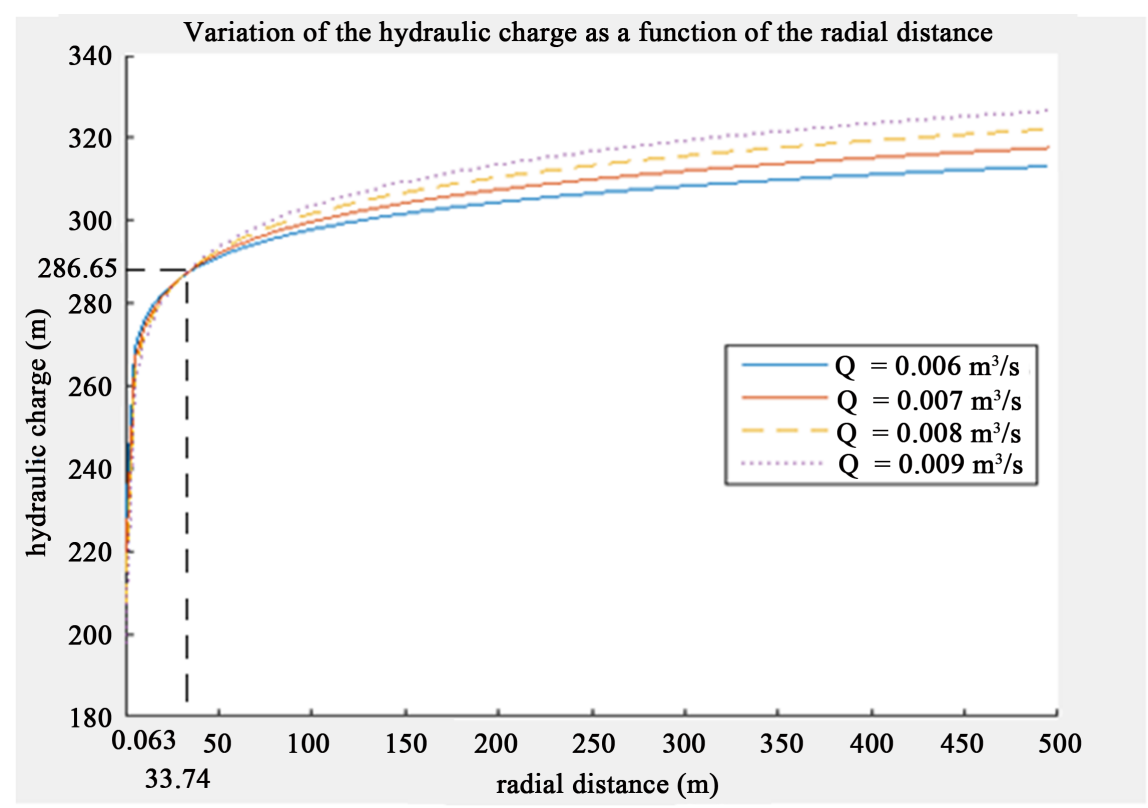

(b)

Figure 3. (a) The hydraulic charge in function of radial distance; (b) The hydraulic charge in function of radial distance.

$$
\left[h-\frac{p_{s t}}{\rho_{w} \cdot g}\right]-\frac{Q}{2 \pi e K}\left[\ln \left(\frac{r}{a}\right)-2 \pi\right]=0, \quad \forall Q \in \mathbb{R}
$$

This equation is equal to zero, if simultaneously, we have:

$$
\left\{\begin{array}{l}
h-\frac{P_{s t}}{\rho_{w} g}=0 \\
\ln \left(\frac{r}{a}\right)-2 \pi=0
\end{array}\right.
$$


thus: $\left\{\begin{array}{l}h=\frac{P_{s t}}{\rho_{w} g} . \\ r=a e^{2 \pi}\end{array}\right.$

Using the following data:

$P_{s t}=28.12$ bars $; \rho_{w}=1000 \mathrm{~kg} / \mathrm{m}^{3} ; g=9.81 \mathrm{~N} / \mathrm{kg} ; \quad a=D / 2=0.063 \mathrm{~m}$

We get: $\left\{\begin{array}{l}h=\frac{28.12 \times 10^{5}}{1000 \times 9.81} \\ r=0.063 e^{2 \pi}\end{array}\right.$.

At least, we obtain a P point $\left\{\begin{array}{l}h=286.65 \mathrm{~m} \\ r=33.74 \mathrm{~m}\end{array}\right.$.

All the curves are logarithmic curves and all go across, at a common point $\mathrm{P}$ $(h=286.65 \mathrm{~m} ; r=33.74 \mathrm{~m}$ ). The curves appear as two parts of the curve in two intervals created by this P point; thus, there are the first interval $0.0063 \otimes r$ $Q 33.74$ and the second one $33.74 \ominus r \otimes R$ where $R$ is the well-range.

In the first interval, no matter the flow rates, all curves get a common part where all the curves are identic and mixed; and in the second one, the curves are different, in accordance to water flowrates.

Observing curves, we note that, the drawdown $(s)$ tends to zero (by adopting as asymptotic direction, the horizontal direction i.e. the static pressure piezometric surface level) while the radial distance tends to well-range. Figure 3 proves that the drawdown occurs significantly in the close proximity of the well, for variable different flowrates. It belongs to the vertical line delimiting the influence zone of the drawdown in two intervals. Thus, the following remarks are:

- In the case of $0.063 \mathrm{~m} \leq r<33.74 \mathrm{~m}$, the vertical asymptotic direction, is the hydraulic charges axis. In this zone, the drawdown is appreciable until the radial distance $r=33.74 \mathrm{~m}$.

- At the P point $(33.74 \mathrm{~m} ; 286.65 \mathrm{~m})$, the drawdown is identic no matter the value of flowrate; when $r=33.74 \mathrm{~m}$, all hydraulic charge curves intersect regardless of the value of the flowrate; thus; at this point, the common optimum value of drawdown $s_{\text {opt }}$ is available.

- When $r>33.74 \mathrm{~m}$, for higher production flow rate, the closer representative hydraulic charge curve is in the undisturbed aquifer charge (initial piezometric surface). The water production does not influence the initial piezometric surface. When the production rate increases, then the corresponding drawdowns are low compared to those of previous flow rates. In the same way, the different drawdowns observed for the different hydraulic charge curves (according to the different flow rates) decrease when the radial distance $(r)$ increases and are cancelled while the radius of action $(R)$ of the well is reached.

\section{Conclusion}

This paper attended to the mathematical steady-state radial modelling simulation of flow around the production well in the confined aquifer of Monzoun- 
goudo. All the curves, obtained for the different flow rates, have as an asymptotic direction, the axis of the hydraulic charges. The variation of the hydraulic charge is a function of the radial distance for different flow rates. The result shows that, on one hand, the drawdown occurs significantly in the close proximity of the well, for variable values of flow; on the other hand, the obtained point $\mathrm{P}$ ( $h=286.65 \mathrm{~m} ; r=33.74 \mathrm{~m})$ is the crossing point of all curves for different production flowrates in the well. It is where we get the optimum of production flowrate for the optimal hydraulic charge in a steady-state flow condition in a confined water table.

\section{Acknowledgements}

This work was supported by ICMPA-UNESCO Chair and LSTE.

\section{Conflicts of Interest}

We, authors declare that we have no competing interests.

\section{References}

[1] Babilas, H. (2018) Hydrodynamique du système Réservoir-Puits de production de Monzoungoudopour son approvisionnement en eau. Thèse de Doctorat de l'Universitéd'Abomey-Calavi, Bénin, 141 p.

[2] Recordon, M.E. (1968) Méthodesmodernes de calcul des débitset des réserves des nappes d'eau souterraines. Bulletin Technique de la Suisse Romande, 94, 7-11.

[3] Laurent, H., Fabris, H. and Gringarten, A.C. (1972) Etude des possibilités exploitation de l'Eau chaude du DOGGER dans la région du CORBEIL-ESSONNES (91). Bureau de Recherches Géologiqueset Minières, Département de Géothermie, Orléans, 41 p.

[4] De Paule, C.F. (1989) Etude analytique des coactions hydrodynamiques et thermodynamique entre les réservoirs, les puits de production et de reinjection géothermiques. Thèse de Doctorat ès Sciences Minières, Université Polytechnique de l'Industrie Lourde Miskolc, Hongrie, 127 p.

[5] De Paule, C.F. and Hountondji, B. (2017) Axisymetric Ascending Flow in Groundwater Production Well of Monzoungoudo, Benin. International Journal of Scientific \& Engineering Research, 8, 774-777. https://www.ijser.org/

[6] Codo, F.P., Adanhounmè, V. and Adomou, A. (2012) Analytical Approach for the Determination of Complex Potential and Pressure in the Production and Reinjection Wells of Geothermal Reservoir. Journal of Applied Sciences Research, 8, 261-266.

[7] Ben, L.M. (2002) Simulation des écoulements dans les puitsproducteurs avec injection. Mémoire de fin d'études d'Ingénieur d'Etat, Université Ouargla d'Algérie, $77 \mathrm{p}$.

[8] Berni, N. and Yacoub, O.E. (2012) Etude numérique d'un écoulement dans un milieu poreux. Mémoire de Master Professionnel, Université Kasdi Merbah Ouarglad' Algérie, $40 \mathrm{p}$.

[9] Ben, A.F. (2005) Analyse des écoulements et du transport de contaminants dans les rejets miniers entreposés dans les massifs rocheux fracturés. Thèse de Doctorat, Ecole Polytechnique de Montréal, 155 p. 


\section{Nomenclature}

a: radius of the well

$R$ : well-range

$r$. radial distance $(a \leq r \leq R)$

$h_{R}$ : hydraulic charge in the aquifer

$h_{W}:$ hydraulic charge in the well

$p$ : pressure

$p_{s i}$ static pressure

$P_{w}$ : bottom pressure of the well

$R_{e}$ : Reynolds number

$h$ : hydraulic charge

$\rho_{w}:$ density of the water

$g$. acceleration of the gravity

$Z_{h}$ : altitude

$K$ : hydraulic conductivity of the medium

$\mu$ : dynamic viscosity of the fluid

$\vartheta$. kinematic viscosity of the fluid

$k$ intrinsic permeability of the medium

$\phi$ : porosity of the medium

$v$ : darcy velocity

$P_{h q}$ hydrodynamic flow pressure,

s. drawdown

$S$ : storage specific coefficient

$H$ : depth of the well

$e$ : productive thickness of sheet

$Q$ : water flowrate

$\lambda$ : coefficient of discharge 